\title{
¿LA ACTITUD ROMÁNTICA HACIA EL AMOR ES LA QUE PREDOMINA CUANDO SE ELIJE PAREJA?
}

\author{
IS THE ROMANTIC ATTITUDE TOWARDS LOVE \\ THE ONE THAT PREVAILS WHEN CHOOSING A MATE?
}

\author{
Kasanzew, A. \\ Universidad Católica de Santa Fe (UCSF) y FUNSALED \\ López Pell, A. F. \\ Universidad Católica de Santa Fe (UCSF) y FUNSALED \\ Brasca, Luciana \\ Universidad Católica de Santa Fe (UCSF) y FUNSALED \\ Fernández, M. S. \\ Universidad Católica de Santa Fe (UCSF) y FUNSALED \\ Prudente, Irene \\ Universidad Católica de Santa Fe (UCSF) y FUNSALED
}

\begin{abstract}
Resumen: Mediante un diseño descriptivo se estudiaron estilos de amor y vínculos de apego romántico en una muestra intencional de alumnos de psicología. El objetivo fue conocer los estilos predominantes mediante las escalas de Actitudes sobre el Amor y Tipos de Apego en contextos románticos. También se recolectó información sobre los vínculos románticos de los participantes. Los resultados muestran una preferencia por el estilo eros de amor, en coincidencia con estudios previos. Sin embargo, el tipo de apego romántico más frecuente fue el temeroso, en clara disidencia a trabajos previos publicados sobre el tema. Entre los resultados obtenidos destaca que el $50 \%$ de la muestra expresó su duda hacia la posibilidad de casarse con una persona que reúna los requisitos añorados pero de la cual no se estuviese enamorado, cuando la negativa hubiese sido la respuesta esperable acorde al estilo de amor predominante.
\end{abstract}

Palabras clave: estilos de amor, vínculos de apego, pareja

Abstract: Following a descriptive design, love styles and romantic attachment bonds were studied in an intentional sample of Psychology students. The target was to know the predominant styles by means of the Love Attitudes Scale and The Attachment Scale in romantic contexts. Information about romantic ties of the participants was also gathered. Results show a preference to Eros style of love, in coincidence with previous studies. Nevertheless, the most frequent romantic attachment type was the fearful one, in clear dissent to previous works published on the topic. Among the obtained results, it is emphasized that $50 \%$ of the sample expressed doubts towards the possibility to marry a person who assembles all the longed requisites but of whom one was not in love - when denial would have been the expected response according to the predominant love style.

Key words: love styles, attachment bonds, couple

Correspondencia: Andrés López Pell. Ph. D; Profesor Titular de la Universidad Católica de Santa Fe. Correo electrónico: alopezpell@funsaled.org.ar 


\section{INTRODUCCIÓN}

El amor y el apego juegan un rol importante dentro de los vínculos románticos adultos. Si bien en sus comienzos la teoría del apego focalizó su atención en niños para el desarrollo de modelos de trabajo del sí mismo en relación con otros significativos (Bowlby, 1980, 1982), en las últimas dos décadas ha extendido su interés por el estudio del apego en díadas adultas (e.g., Hazan \& Shaver, 1994; Shaver, Hazan \& Bradshaw, 1988). De acuerdo a la evidencia, pareciera que la calidad de los cuidados parentales que el infante recibe en sus primeros años no sólo es de vital importancia para su bienestar psicológico sino que también determinan, en cierta forma, la cualidad de los vínculos de pareja en su adultez.

\section{EL MODELO DE LEE $(1973,1988)$ DEL AMOR}

La psicología ha sorteado la dificultad de dar una definición conceptual del amor al postular diferentes clasificaciones o tipologías (véase Dion \& Dion, 1973; Hazan \& Shaver, 1987; Hendrick \& Hendrick, 1986; Lee, 1973; Rubin, 1973; Sternberg, 1986). Una tipología muy utilizada en investigación sobre el amor es la propuesta de Lee (1973), quien a partir de una analogía con los colores diferenció seis estilos de amor preponderantes, a los que dividió en primarios y secundarios. Entre los estilos primarios se encuentran Eros, Storge y Ludus $y$, como consecuencia de combinar estos tres, aparecen tres estilos secundarios: Manía, Pragma y Ágape. Según las descripciones de Lee $(1973 ; 1988)$ el primer estilo, Eros, está representado por el amor romántico y pasional en donde se sabe con claridad qué atributos físicos gustan en una potencial pareja, a quien desea conocer de forma rápida e intensa. El segundo estilo, Storge, está representado por un amor afectuoso y comprometido que se desarrolla sin prisa a partir de una sólida amistad. Ludus, por su parte, describe al amante juguetón, quien no tiene en mente ningún ideal de pareja, no busca compromisos, es pluralista y nada celoso. Por el contrario, el estilo Manía representa al amor obsesivo, celoso y posesivo, el cual necesita que la pareja de cuenta de su compromiso de forma constante. El quinto estilo, Pragma, se caracteriza por ser lógico y pragmático: lleva en mente una lista de cualidades prácticas que desea en una pareja, quien tendrá que ser compatible en cuestiones como la religión, la política, el nivel social, el hobby o el deporte. El sexto y último estilo, Ágape, es un amor dador, autosacrificado y altruista que carece de egoísmo.

Lee (1988) no intenta definir el amor en sí mismo, sino describir cómo se aman dos personas. Es decir, el estilo no define al amante sino a la relación. Más aún, sostiene que un sujeto puede cambiar su preferencia por un estilo u otro durante su vida o, incluso, mantener dos estilos a la vez con parejas diferentes. De esta manera, asume la posibilidad tanto de aprender como de cambiar diferentes estilos de amor de acuerdo a la experiencia romántica que se juega en una relación dada.

Hendrick y Hendrick (1986) idearon la Escala de Actitudes hacia el Amor para evaluar los seis estilos desde una perspectiva individual de las relaciones amorosas, herramienta que le ha dado validez teórica a la propuesta de Lee (1973). En los trabajos de Hendrick y Hendrick (1986) con estudiantes de Miami, de Zubieta (2001) en población española y de Brenlla, Brizzio y Carreras (2003) en Argentina, los resultados mostraron que tanto hombres como mujeres privilegian los estilos Eros y Ágape de amor.

\section{DESARROLLOS SOBRE EL APEGO DESDE LA INFANCIA A LA PAREJ A ADULTA}

Según Bowlby (1980, 1982), el apego refiere a cualquier conducta de un individuo que tiene como objetivo alcanzar o mantener un contacto cercano con otra persona, usualmente más fuerte o sabia, la cual es reconocida como una figura de apego. Cuando esta proximidad sufre alguna interrupción, la separación es vivida con algún grado de estrés o protesta. Otra finalidad del apego en tiempos de peligro o amenaza es que opera como un refugio seguro, donde la figura de apego brinda seguridad, protección y apoyo. Por último, un tercer objetivo del apego será utilizar la figura de apego como una base segura desde donde salir a explorar el mundo. A medida que las conductas de apego se repiten, el infante internaliza y organiza su experiencia de apego en un sistema de representaciones sobre el sí mismo en relación con las figuras de apego. Este proceso da lugar a la formación de modelos operativos de trabajo o working models, los cuales estarán basados 
en dos criterios: a) si se representa la figura de apego como una persona que en general responderá a las solicitudes de apoyo y protección; y b) si el infante se juzga a sí mismo como una persona digna de recibir o no tal apoyo (Fraley \& Shaver, 2000). Si el infante puede contar con un cuidador principal previsiblemente disponible, cariñoso, tolerante y empático, tendrá entonces mayor probabilidad de desarrollar un modelo operativo interno que pueda ser descrito como seguro. Por el contrario, si el niño o la niña crece con una figura parental poco previsible y responsiva, es más probable que desarrolle un estilo de apego inseguro, ya que la función primaria de la figura de apego es la de brindar protección en situaciones que de miedo o ansiedad. Ainsworth et al. (1978) profundizaron el trabajo de Bowlby $(1980,1982)$ y llevaron a cabo un estudio sobre las diferencias individuales en el apego entre el infante y su madre a través de un procedimiento de laboratorio denominado la situación extraña, que les permitió identificar y describir tres estilos de apego: a) inseguro evitativo: el infante se muestra desapegado y evita el contacto con su cuidador; b) seguro: el infante muestra una mayor habilidad para jugar y explorar el ambiente con alegría, seguridad y curiosidad; una mayor capacidad para mostrar disgusto como una reacción apropiada a la separación; y una mayor capacidad para ser calmado por su cuidador; y c) inseguro ambivalente o resistente: el infante muestra estrés frente a la separación y conductas de protesta y enojo ante el reencuentro, no se calma con facilidad y no retoma la actividad de exploración. Los estilos inseguros ambivalente y evitativo se producen frente a una pérdida prematura o ausencia prolongada de la madre o su sustituto, tiempo durante el cual el infante no recibe cuidados y protección. Esto provoca la situación Ilamada privación materna, la cual puede generar en el niño estilos de apego inseguros (Ainsworth et al., 1978). Por otro lado, si el infante siente que sus figuras de apego son fiables y están disponibles en forma constante, es más probable que explore el mundo que lo rodea, ya que supondrá que estas figuras estarán allí cuando decida regresar. Esta experiencia es la que Ainsworth et al. (1978) denominaron usar a la madre como base segura desde donde explorar.

El estudio del apego adulto se fundamenta en la hipótesis de que los patrones de apego establecidos al comienzo de la vida son relativamente estables a través del desarrollo, y que los vínculos de apego diádicos son el prototipo del apego adulto (Hazan \& Zeifman, 1999). Como en una pareja adulta ambos integrantes son proveedores de seguridad cualidad princeps del apego- los autores sostienen que "las relaciones románticas califican como vínculos de apego y por lo tanto constituyen el contexto apropiado para investigar el fenómeno del apego adulto" ( $p$. 336). Una de las diferencias entre el apego entre infante y cuidador y entre adultos es en el primer caso las funciones son complementarias, mientras que en el segundo son simétricas e intercambiables. Por otro lado, si bien el contacto físico es otra cualidad propia tanto de la díada infante-cuidador como de la relación de pareja, la diferencia está dada en que en la última entra en juego el sexo (Hazan \& Shaver, 1994; Shaver, Hazan \& Bradshaw, 1988). Hazan y Shaver (1987) adoptaron la tipología de Ainsworth et al. (1978) como marco para organizar las diferencias individuales en la manera en cómo los adultos piensan, sienten y se comportan en las relaciones románticas. De esta manera, describieron tres tipos cualitativamente distintos de apego romántico: a) seguro: muestran auto-confianza, habilidades sociales, apertura e interés en las relaciones íntimas, satisfacción y estabilidad en las relaciones a largo plazo, tienden a vivir las relaciones afectivas con alegría y emociones positivas, confían en el otro, aceptan en general a su pareja a pesar de sus defectos y tienen mayor capacidad para resolver los conflictos interpersonales; b) ansioso-ambivalente: personas con poca auto confianza, preocupadas por el castigo y el abandono, celosas y reacias a tener relaciones románticas a largo plazo y con una mayor vulnerabilidad a la soledad; y c) evitativo: sujetos que se muestran más o menos interesados en relaciones íntimas, pero que muestran poca disposición para mantener relaciones románticas a largo plazo, inhibición social, rechazo a la intimidad, dificultad para depender de los demás, falta de confianza, altibajos emocionales y dificultad para aceptar los defectos de la pareja (Brennan \& Shaver, 1995; Feeney \& Noller, 1990; Hazan \& Shaver, 1987; Pistole, 1995).

El apego adulto se ha evaluado a través de diferentes instrumentos (e.g., Brenlla, Brizzio 
\& Carreras, 2003; Griffin \& Bartholomew, 1994; Pottharst, 1990) y ha mostrado una preferencia mayoritaria por el tipo seguro. En Argentina, Casullo (2002) diseñó una nueva escala que, luego de haber sido sometida a un análisis psicométrico (Casullo \& Fernández Liporace, 2005), arrojó la presencia de tres factores: a) temeroso-evitativo; b) ansioso; y c) seguro, estructura factorial que resultó coherente con la propuesta de Ainsworth, Blehar, Waters y Wall (1978) y la de Hazan y Shaver (1987) de tres estilos de apego. La adaptación argentina de la escala de amor como la de apego se aplicaron sólo una vez en población general (Brenlla et al., 2003; Casullo, 2002) y una vez en población homosexual adulta (Kasanzew, 2007).

Por todo lo enunciado anteriormente es que se entiende la pertinencia de estudiar el estilo de amor y el tipo de apego romántico predominante en distintas poblaciones. Esta necesidad se apoya en dos hechos: a) tanto la Escala de Actitudes sobre el Amor como la de Apego en contextos románticos son instrumentos relativamente nuevos $y, b$ ) no han sido aún vastamente utilizados en población de habla hispana. En relación a los trabajos previos con estas técnicas (e.g., Brenlla et al., 2003; Casullo, 2002; Kasanzew, 2007) es que se propuso verificar: a) si los resultados de este trabajo eran similares a los de las investigaciones anteriores, b) si las variables sociodemográficas recolectadas arrojaban alguna diferencia significativa.

\section{MÉTODO}

Se eligió un diseño descriptivoexploratorio, en el cual participaron en forma voluntaria un total de 68 estudiantes de psicología de ambos sexos, quienes fueron elegidos por disponibilidad. La muestra intencional estuvo compuesta por sujetos de entre 21 y 49 años de edad, residentes en la Ciudad de Santa Fe.

Para evaluar las variables principales se utilizaron dos escalas autoadmnistrables. Para estudiar los estilos de amor de Lee (1973) se aplicó la Escala de Actitudes sobre el Amor (ASA) de Hendrick y Hendrick (1986), adaptada a población argentina por Casullo (2001). El instrumento consta de 42 ítemes, los cuales son evaluados a través de una escala tipo Likert de cinco puntos. Por otro lado, para conocer el apego romántico adulto predominante, los participantes respondieron la Escala de Apego (Casullo, 2002). La herramienta se compone de 9 reactivos, los cuales se puntúan en una escala tipo Likert de cuatro opciones.

Asimismo, se recolectó información sobre el sexo, edad, nivel de estudios, empleo y lugar de residencia de los sujetos. En cuanto a las relaciones románticas, se preguntó si estaban en pareja al momento de responder la encuesta, cuántas veces han estado enamorados, cuántas veces han estado involucrados en una pareja romántica y cuántas parejas casuales han tenido.

Por último, se planteó la siguiente situación: ¿Si una persona tuviese todas las características que usted considera deseables, formaría una pareja con ella aunque no estuviese enamorado/a?, con tres opciones de respuesta: No, Sí y No lo sé.

\section{RESULTADOS}

La muestra intencional estuvo conformada por alumnos de ambos sexos que cursaban el cuarto de año de la carrera de psicología en la Universidad Católica de Santa Fe ( $\mathrm{N}=68)$, quienes participaron de forma voluntaria y anónima. La media de edad fue de 23 años (d.t., $3,35)$, con una mínima de 21 y una máxima de 36 años. Catorce personas pertenecían al sexo masculino y 54 al sexo femenino, más del $90 \%$ residía en la Ciudad de Santa $\mathrm{Fe}$, el 95\% tenía estudios universitarios incompletos y más del $75 \%$ sólo estudiaba.

Los participantes informaron estar enamorados un máximo de 5 veces $(\mathrm{M}=2,0$; d.t. $=1,193)$, haber tenido hasta diez parejas románticas $(\mathrm{M}=2,68$; d.t. $=2,156)$ y 50 casuales $(M=5,42 ;$ d.t. $=11,345)$. De los 68 participantes, casi el $60 \%$ informó estar en pareja y cerca del $40 \%$ no formaría una relación romántica sin estar enamorado.

\section{LOS VÍNCULOS DE APEGO ROMÁNTICO}

Con el objetivo de conocer cuál es tipo de apego romántico que caracteriza más a los estudiantes de la muestra, se calculó la prueba t para una muestra. Los resultados revelan que el tipo de apego más característico es el temeroso, seguido por el seguro y el evitativo (véase Tabla 1). 
Diferencias en los vínculos de apego romántico

Para evaluar la presencia de diferencias en los vínculos de apego según el sexo de los participantes (i.e., masculino y femenino) se utilizó la prueba t para muestras independientes. Como se observa en la tabla 2 , se encontró una diferencia estadísticamente significativa a favor del grupo integrado por el sexo masculino, el cual mostró un apego más temeroso que las participantes del sexo femenino $(p<.05)$.

Asimismo, se evaluaron diferencias en los vínculos de apego según si los participantes estaban o no en pareja y según las respuestas a la pregunta: ¿Si una persona tuviese todas las características que desea, formaría una pareja aunque no estuviera enamorado?, pero no se encontraron diferencias estadísti- camente significativas en ninguno de los casos $(p<.05)$.

\section{LOS ESTILOS DE AMOR}

Con el objetivo de conocer cuál es tipo de amor que caracteriza más a los estudiantes de la muestra, se calculó la prueba t para una muestra. Los resultados revelan que el tipo de amor más característico es Eros, seguido por Ágape, Manía, Pragma, Storge y Ludus respectivamente (véase Tabla 3 ).

Diferencias en los estilos de amor

Para verificar diferencias en los estilos de amor según si se está o no en pareja, se utilizó la prueba t para muestras independientes. Como muestra la tabla 4 , se verificó una diferencia

Tabla 1. Diferencia de medias para los estilos de apego $(\mathrm{N}=68)$.

\begin{tabular}{lcccc}
\hline \multicolumn{1}{c}{ Tipo de apego } & $\mathbf{T}$ & $\mathbf{g l}$ & Sig. & Dif. de medias \\
\hline Seguro & 16,855 & 33 &, 000 & 3,88 \\
Ansioso & 12,138 & 33 &, 000 & 2,94 \\
Temeroso & 20,205 & 33 &, 000 & 8,35 \\
\hline
\end{tabular}

Tabla 2. Vínculos de apego romántico y sexo

\begin{tabular}{|c|c|c|c|c|c|c|c|}
\hline Tipo de apego & gl & $\mathbf{F}$ & $p$ & Sexo & $\mathbf{N}$ & $M$ & d.t. \\
\hline \multirow{2}{*}{ Seguro } & 66 & 1,505 & n.s. & Masculino & 14 & 4,29 & 1,704 \\
\hline & & & & Femenino & 54 & 3,78 & 1,251 \\
\hline \multirow[t]{2}{*}{ Ansioso } & 66 & 951 & n.s. & Masculino & 14 & 2,71 & ,756 \\
\hline & & & & Femenino & 54 & 3,00 & 1,544 \\
\hline \multirow[t]{2}{*}{ Temeroso } & 66 & 166 &, $027^{* *}$ & Masculino & 14 & 9,14 & 2,410 \\
\hline & & & & Femenino & 54 & 8,15 & 2,413 \\
\hline
\end{tabular}

Tabla 3. Diferencia de medias para los estilos de amor $(\mathrm{N}=68)$.

\begin{tabular}{lcccc}
\hline \multicolumn{1}{c}{ Estilo de amor } & $\mathbf{T}$ & gl & Sig. & Dif. de medias \\
\hline Eros & 57,945 & 67 &, 000 & 26,59 \\
Ludus & 26,009 & 67 &, 000 & 17,94 \\
Storge & 22,690 & 67 &, 000 & 18,65 \\
Pragma & 24,264 & 67 &, 000 & 19,35 \\
Manía & 20,472 & 67 &, 000 & 20,79 \\
Ágape & 36,819 & 67 &, 000 & 24,38 \\
\hline
\end{tabular}

Tabla 4. Estilos de amor y situación de pareja

\begin{tabular}{|c|c|c|c|c|c|c|c|}
\hline Estilo de amor & $\mathrm{gl}$ & $\mathbf{F}$ & $p$ & En pareja & $\mathbf{N}$ & $\mathbf{M}$ & d.t. \\
\hline Eros & 66 & 1,177 & n.s. & $\begin{array}{l}\text { SI } \\
\text { NO }\end{array}$ & $\begin{array}{l}40 \\
28\end{array}$ & $\begin{array}{l}26,70 \\
26,43\end{array}$ & $\begin{array}{l}2,940 \\
2,344\end{array}$ \\
\hline Ludus & 66 & 2,300 & n.s. & $\begin{array}{l}\mathrm{SI} \\
\mathrm{NO}\end{array}$ & $\begin{array}{l}40 \\
28\end{array}$ & $\begin{array}{l}16,95 \\
19,36 \\
\end{array}$ & $\begin{array}{l}3,268 \\
4,668\end{array}$ \\
\hline Storge & 66 & ,362 &, $009^{*}$ & $\begin{array}{l}\text { SI } \\
\text { NO }\end{array}$ & $\begin{array}{l}40 \\
28\end{array}$ & $\begin{array}{l}16,90 \\
21,14\end{array}$ & $\begin{array}{l}4,723 \\
3,780\end{array}$ \\
\hline Pragma & 66 & ,785 & n.s. & $\begin{array}{l}\mathrm{SI} \\
\mathrm{NO}\end{array}$ & $\begin{array}{l}40 \\
28\end{array}$ & $\begin{array}{l}18,85 \\
20,07\end{array}$ & $\begin{array}{l}5,224 \\
3,751\end{array}$ \\
\hline Manía & 66 & 2,019 & n.s. & $\begin{array}{l}\mathrm{SI} \\
\mathrm{NO}\end{array}$ & $\begin{array}{l}40 \\
28\end{array}$ & $\begin{array}{l}21,80 \\
19,36 \\
\end{array}$ & $\begin{array}{l}4,884 \\
7,099\end{array}$ \\
\hline Ágape & 66 & ,061 & n.s. & $\begin{array}{l}\mathrm{SI} \\
\mathrm{NO}\end{array}$ & $\begin{array}{l}40 \\
28 \\
\end{array}$ & $\begin{array}{l}24,75 \\
23,86 \\
\end{array}$ & $\begin{array}{l}3,919 \\
3,860 \\
\end{array}$ \\
\hline
\end{tabular}

${ }^{*} p<.01$ 
estadísticamente significativa $(p<.01)$ a favor del estilo Storge, el cual fue más apoyado por los sujetos que no están en una relación romántica que por las personas que informaron estar en pareja.

Por otro lado, no se verificaron diferencias significativas $(p<.05)$ en los estilos de amor según la variable sexo (i.e., masculino y femenino) y según las respuestas a la pregunta: ¿Si una persona tuviese todas las características que desea, formaría pareja aunque no estuviera enamorado?

Por último, se destaca que no se encontraron asociaciones positivas o negativas entre los estilos de amor y los tipos de apego.

\section{CONCLUSIÓN}

La muestra estuvo representada por estudiantes sin empleo del cuarto año de la carrera de Psicología de la Universidad Católica de Santa Fe, quienes informaron haber estado enamorados al menos dos veces, haber tenido casi tres parejas románticas y algo más de cinco casuales al momento de participar de la investigación ( $M=23$ años; d.t.=3,35). Además, casi el $50 \%$ de los sujetos no está seguro si se casaría sin estar enamorado, a pesar de que la pareja reúna todas las características deseadas.

Al igual que las muestras de los estudios de Brenlla et al. (2003), Hendrick y Hendrick (1986) y Zubieta (2001), los estudiantes de psicología de Santa Fe presentan una alta adhesión al estilo Eros de amor: saben qué tipo de físico los atrae y qué atributos quieren en una potencial pareja, a la vez que desean vivir la experiencia romántica con pasión, intensidad y exclusividad. A este estilo no posesivo ni celoso de amar, los sujetos lo refuerzan con la preferencia por el estilo agápico, es decir, un amor carente de egoísmo, dador, altruista, gentil, paciente y auto sacrificado, guiado más por las necesidades del otro que por las propias. Por otro lado, muestran un alto desacuerdo por un amor pragmático; o sea, no buscan una pareja por conveniencia o con ciertos atributos específicos (e.g., buen estatus social y económico). En consonancia con la preferencia por el estilo Eros, no se comparte la idea del amor como una amistad que se desarrolla sin la fiebre de la pasión. No obstante, aquellos que informaron no estar en pareja prefieren significativamente más este estilo amistoso que quienes están en una relación romántica.

Por último, la alta adhesión por el estilo ansioso temeroso de apego en contextos románticos describe a los estudiantes de la muestra con poca auto confianza, preocupados por el abandono y celosos. Si bien buscan la unión y la reciprocidad en sus vínculos románticos adultos, la experiencia de estas relaciones se caracteriza por preocupación obsesiva, desconfianza y un sentimiento de vulnerabilidad frente a la soledad. El estilo ansioso temeroso de los estudiantes de la muestra no les permitiría la formación de apegos íntimos y de confianza, ni usar a la pareja como una base segura desde donde salir a explorar el mundo de las relaciones interpersonales. Por otro lado, la presencia del estilo ansioso temeroso en los vínculos románticos indicaría que durante la primera infancia no han tenido experiencias satisfactorias con sus cuidadores primarios, quienes probablemente no hayan estado disponibles en tiempos de necesidad y peligro (Ainsworth et al., 1978; Bowlby, 1982). Por último, se destaca como diferencia entre los sexos que los varones de la muestra se mostraron más temerosos que las mujeres.

\section{DISCUSIÓN}

La alta adhesión que obtuvo el estilo Eros de amor es congruente con lo informado por estudios previos y claramente parece mostrar una elección en sintonía con la idea de amor romántico que sostiene aún la cultura occidental. Ahora bien, en contraposición con este ideal de amor, los estudiantes se muestran temerosos a la hora de confiar en sus parejas. Estos resultados podrían estar indicando una brecha significativa entre lo que se cree debería ser el amor y lo que finalmente queda plasmado en la realidad de los vínculos afectivos adultos. Asimismo, el hecho de que casi la mitad de la muestra dudara si formaría pareja sin estar enamorado a pesar del alto apoyo dado a eros, argumenta relativamente a favor de la posibilidad que tiene el sujeto de cambiar, tal como postula Lee $(1973 ;$ 1988), las concepciones sobre las relaciones románticas y pasar, por ejemplo, de un estilo Eros a otro pragmático de amor de acuerdo a cómo se define un vínculo en particular.

Por último, la mayor adhesión de los varones frente a las mujeres por el tipo de apego temeroso, ofrecería cierta evidencia sobre una mayor dificultad del género masculino para usar al otro como una figura de apego en momentos de estrés o peligro. 


\section{REFERENCIAS}

Ainsworth, M. D. S., Blehar, M., Waters, E. \& Wall, S. (1978). Patterns of attachment. Hillsdale, NJ: Erlbaum.

Bowlby, J. (1980). Attachment and loss. Vol. 3. Loss: Sadness and depression. New York: Basic Books.

Bowlby, J. (1982). Attachment and loss. Vol. 1. Attachment (2a. ed.). New York: Basic Books.

Brenlla, M. E., Brizzio, A. \& Carreras, A. (2003). Actitudes hacia el amor y apego. Psicodebate, 4, 7-23.

Brennan, K. A. \& Shaver, P. R. (1995). Dimensions of adult attachment, affect regulation, and romantic relationship functioning. Personality and Social Psychology Bulletin, 21, 267-283.

Casullo, M. M. (2001). Actitudes sobre el amor. Publicación. Facultad de Psicología. Universidad de Buenos Aires.

Casullo, M. M. (2002). Escalas para la evaluación de estilos de apego. Publicación. Facultad de Psicología: Universidad de Buenos Aires.

Casullo, M. M. \& Fernández Liporace, M. (2005). Los estilos de apego: Teoría y medición. Buenos Aires: JVE.

Feeney, J. A. (1999). Adult romantic attachment and couple relationships. En J. Cassidy y P. R. Shaver (Eds.), Handbook of attachment: Theory, research, and clinical applications (pp. 355-377). New York: Guildford Press.

Feeney, J. A. \& Noller, P. (1990). Attachment style as a predictor of adult romantic relationships. Journal of Personality and Social Psychology, 58, 281-291.

Fraley, R. C. \& Shaver, P. R. (2000). Adult Romantic Attachment: Theoretical Developments, Emerging Controversies, and Unanswered Questions. Review of General Psychology, 4, 132-154.

Griffin, D. W. \& Bartholomew, K. (1994). Models of the self and other: Fundamental dimensions underlying measures of adult attachment. Journal of Personality and Social Psychology, 67, 430-445.

Hazan, C. \& Shaver, P. R. (1987). Romantic love conceptualized as an attachment process.
Journal of Personality and Social Psychology, 52, 511-524.

Hazan, C. \& Shaver, P. R. (1994). Attachment as an organizational framework for research on close relationships. Psychological Inquiry, 5, 1-22.

Hazan, C. \& Zeifman, D. (1999). Pair bonds as attachments: Evaluating the evidence. En J. Cassidy y P. R. Shaver (Eds.), Handbook of attachment: Theory, research, and clinical applications (pp. 336354). New York: Guildford Press.

Hendrick, C. \& Hendrick, S. S. (1986). A theory and method of love. Journal of Personality and Social Psychology, 50, 392-402.

Kasanzew, A. (2007). Criterios de elección de pareja, vínculos de apego y estilos de amor en varones homosexuales adultos argentinos. Tesis Doctoral. Buenos Aires: Universidad de Palermo.

Sternberg, R. J. (1986). A triangular theory of love. Psychological Review, 93, 119-135.

Lee, J. A. (1973). The colors of love: An exploration of the ways of loving. Toronto: New Press.

Lee, J. A. (1988). Love-styles. En R. J. Sternberg y M. L. Barnes (Eds.), The psychology of love (pp. 38-67). New Haven: Yale University Press.

Pistole, M. C. (1995). College students' ended love relationships: Attachment style and emotion. Journal of College Students Development, 1, 53-60.

Pottharst, K. (1990). Explorations in adult attachment. New York: Peter Lang.

Rubin, Z. (1973). Liking and loving: An invitation to social psychology. New York: Holt, Rinehart, y Winston.

Sternberg, R. J. (1986). A triangular theory of love. Psychological Review, 93, 119-135.

Shaver, P. R., Hazan, C. \& Bradshaw, D. (1988). Love as attachment: The integration of three behavioral systems. En R. J. Sternberg y M. L. Barnes (editores), The psychology of love (pp.68-99). New Haven: Yale University Press.

Zubieta, E. M. (2001). Actitudes sobre el amor y preferencias en la elección de pareja. Buenos Aires: Facultad de Psicología. 